TITLE PAGE

\title{
MASKING OF THRESHOLDS FOR THE PERCEPTION OF FORE-AND-AFT VIBRATION OF SEAT BACKRESTS
}

Authors:

Miyuki Morioka and Michael J. Griffin

Affiliations:

Human Factors Research Unit

Institute of Sound and Vibration Research

University of Southampton

Southampton, SO17 1BJ

England

\section{Corresponding author:}

Miyuki Morioka

Human Factors Research Unit

Institute of Sound and Vibration Research

University of Southampton

Southampton, SO17 1BJ

England

Email: M.Morioka@soton.ac.uk (correspond to the first author)

Tel: +44 (0)2380 593723 or +44 (0)2380 592277

Number of pages: $\quad 25$

Number of figures: $\quad 8$

Number of tables: $\quad 1$ 


\section{ABSTRACT}

The detection of a vibration may be reduced by the presence of another vibration: a phenomenon known as 'masking'. This study investigated how the detection of one frequency of vibration is influenced by vibration at another frequency. With nine subjects, thresholds for detecting fore-and-aft backrest vibration were determined (for 4, 8, 16, and $31.5-\mathrm{Hz}$ sinusoidal vibration) in the presence of a masker vibration (4- $\mathrm{Hz}$ random vibration, 1/3-octave bandwidth at six intensities). The masker vibration increased thresholds for perceiving vibration at each frequency by an amount that reduced with increasing difference between the frequency of the sinusoidal vibration and the frequency of the masker vibration. The 4-Hz random vibration almost completely masked $4-\mathrm{Hz}$ sinusoidal vibration, partially masked $8-$ and $16-\mathrm{Hz}$ vibration, and only slightly masked $31.5-\mathrm{Hz}$ vibration. The findings might be explained by the involvement of different sensory systems and different body locations in the detection of different frequencies of vibration.

(150 words)

Key words: perception, whole-body vibration, masked threshold 


\section{INTRODUCTION}

Fore-and-aft vibration of a backrest is one of the principal sources of discomfort for drivers and passengers in vehicles. For seated people supported by an upright backrest, equivalent comfort contours for vibration applied to the back show greater sensitivity to fore-and-aft vibration than vibration in either of the other two directions (i.e., vertical or lateral) over the frequency range 2.5 to $63 \mathrm{~Hz}$ (Parsons et al., 1982). Over the range from 2 to $16 \mathrm{~Hz}$, thresholds for perceiving fore-and-aft vibration at the back are similar to, or lower than, thresholds for perceiving vibration at the feet or the seat (Gallais et al., 2014). Since backrests amplify some frequencies of vibration (Jalil and Griffin, 2007; Basri and Griffin, 2014), the perception of fore-and-aft vibration can be dominated by vibration of the back.

Sensitivity to fore-and-aft vibration of the back depends on the frequency of vibration and contact conditions with the backrest. A frequency weighting for evaluating the severity of fore-and-aft backrest vibration (weighting $W_{c}$ in BS 6841:1987 and ISO 2631-1:1997) was based on experimental studies that determined the acceleration of a backrest required at various frequencies to cause discomfort equivalent to that caused by $10-\mathrm{Hz}$ vertical vibration of a seat at a magnitude of $0.8 \mathrm{~ms}^{-2}$ r.m.s. The experiment produced an equivalent comfort contour showing greatest sensitivity to acceleration at frequencies between 2.5 and $8 \mathrm{~Hz}$. At higher frequencies, sensitivity to acceleration reduced in proportion to the frequency of vibration, corresponding to similar discomfort with similar vibration velocity (Parsons et al., 1982). Similar equivalent comfort contours have been reported from other studies with foreand-aft vibration of a full upright backrest (Kato and Hanai, 1998; Morioka and Griffin, 2010; Basri and Griffin, 2011) but with variations dependent on the location of contact with the back (Morioka and Griffin, 2010) and the inclination of the backrest (Kato and Hanai, 1998; Basri and Griffin, 2011). Unlike equivalent comfort contours for vertical seat vibration, it seems that equivalent comfort contours for the back are not highly dependent on the magnitude of vibration (Morioka and Griffin, 2010; Basri and Griffin, 2011). 
The detection of one type of vehicle oscillation may be expected to be influenced by the presence of other vibrations (e.g., background vibration): according to a phenomenon known as 'masking' (i.e., the detection of one stimulus is 'masked' by the excitation associated with another stimulus). With vibrotactile stimuli applied to a small area of the thenar eminence of the hand, masking occurs when the masker stimulus and the test stimulus stimulate the same tactile channel (e.g., Gescheider et al., 1982) and similar masking has been observed in the perception of hand-transmitted vibration (Morioka and Griffin, 2005). There is no known study of the influence of masking on the perception of whole-body vibration. It seems reasonable to anticipate that greater understanding of masking will improve the prediction of the sensations caused by vibration in vehicles.

A 'masked threshold' is the threshold for the perception of a stimulus determined in the presence of another stimulus. It may be expected that masking will be greatest when the two stimuli are perceived by the same mechanism (e.g., by exciting the same sensory system at the same location in the body). This is sometimes called 'energetic masking' (EM), as opposed to 'informational masking' (IM) where the detection of one stimulus is impeded by distraction from another stimulus (Durlach, et al. 2003). When two vibration stimuli have different frequencies, they may be perceived at different body locations even if they enter the body at the same location (because different frequencies excite different motions in the body). For each of the three translational axes of vibration of a seat with no backrest, the location of discomfort in the body is more dependent on the frequency of vibration than the magnitude of the vibration (Whitham and Griffin, 1978). With fore-and-aft vibration of the back in the frequency range 2.5 to $25 \mathrm{~Hz}$, discomfort is mostly localised in the upper or lower back at all frequencies, although with increased perception at the head, neck, or shoulders at 20 and $25 \mathrm{~Hz}$ (Basri and Griffin, 2011). In part, this reflects the complex biodynamic responses of the body to vibration applied at the back (Jalil and Griffin, 2008).

This laboratory study was designed to investigate masked thresholds for sinusoidal fore-andaft vibration of a backrest. It was hypothesised that thresholds for detecting a sinusoidal 
vibration stimulus would increase with increasing intensity of a masker stimulus (a random background vibration) but that the threshold shift would depend on the frequency of the sinusoidal stimulus, reflecting the detection of the different frequencies of vibration either by different sensory systems (e.g. somatosensory, vestibular, visual, etc.) or at different body locations. The findings of this study have application to predicting whether a particular vibration of a vehicle will be detected by drivers or passengers when there are other vibrations present.

\section{METHOD}

\subsection{Subjects}

Nine males aged between 21 and 29 years, with a mean age of 24.6 years (standard deviation, $\mathrm{SD}=2.7)$, a mean stature of $174.7 \mathrm{~cm}(\mathrm{SD}=10.8)$ and a mean weight of $68.8 \mathrm{~kg}$ $(S D=14.1)$ participated in the experiment. All subjects were students or office workers with no history of occupational exposure to vibration.

The experiment was approved by the Human Experimentation Safety and Ethics Committee of the Institute of Sound and Vibration Research at the University of Southampton. Informed consent to participate in the experiment was given by all subjects.

\subsection{Apparatus}

Fore-and-aft backrest vibration was produced by a rigid flat vertical wooden plate $(640 \times 680$ $\mathrm{mm}$ ) secured to a trunnion-mounted Derritron VP 85 vibrator. Subjects sat on a stationary rigid seat and supported their feet on a stationary rigid footrest (Figure 1). The height of the footrest was adjustable so that the upper surfaces of the upper legs of subjects were horizontal.

A piezoelectric accelerometer (DJ Birchall, type A/20T) was attached to the centre of the rear surface of the wooden plate and the signal amplified by a charge amplifier (Brüel and Kjær, type 2635). Vibration signals were generated and acquired using HVLab Data 
Acquisition and Analysis Software (version 3.81) to a personal computer via anti-aliasing filters (TechFilter) and analogue-to-digital and digital-to-analogue converters (PCL-818). The signals were generated at 500 samples per second and passed through $45-\mathrm{Hz}$ low-pass filters. The stimulus parameters and the psychophysical measurement procedures were controlled by the computer. The background vibration, mostly electrical noise at $50 \mathrm{~Hz}$, was less than $0.005 \mathrm{~ms}^{-2}$ r.m.s., and was not perceptible via the backrest. With all stimuli, the cross-axis accelerations were less than $5 \%$ of the fore-and-aft acceleration.

During the experiment, the subjects were exposed to acoustic white noise at $65 \mathrm{~dB}(\mathrm{~A})$ via a pair of headphones so that they all experienced the same noise in all four sessions, so that they could not hear the vibration, and so that they could concentrate on the vibration without being distracted by sounds in the laboratory. The acoustic noise generated by the vibration stimuli was inaudible even without the masking noise.

\section{FIG. 1 ABOUT HERE}

\subsection{Stimuli}

Sinusoidal vibratory stimuli, $2 \mathrm{~s}$ in duration, with rise and fall times of $0.5 \mathrm{~s}$ were created with cosine-tapered ends. Four test stimuli, with frequencies at $4,8,16$, and $31.5 \mathrm{~Hz}$, were prepared. A Gaussian random masking stimulus, $7 \mathrm{~s}$ in duration, was created with a 1/3octave bandwidth centred at $4 \mathrm{~Hz}$ (filter pass-band 3.55 to $4.47 \mathrm{~Hz}$ ). The masking stimulus, which varied in intensity according to the perception threshold for the masker measured for each subject at the beginning of each session, was presented at five levels from threshold up to $24 \mathrm{~dB}$ SL in $6 \mathrm{~dB}$ steps (i.e., at 0, 6, 12, 18, and $24 \mathrm{~dB}$ above the threshold level of the subject). The stimulus parameters, including the combination of test and masker stimulus, are shown in Table 1.

TABLE 1 ABOUT HERE 


\subsection{Procedure}

Subjects attended four sessions (performed on separate days), each corresponding to one of the four test frequencies (i.e., 4, 8, 16, or $31.5 \mathrm{~Hz}$ ). Each session lasted approximately one hour and comprised two parts:

Part A (thresholds for the sinusoidal test stimulus and the random masker)

Part B (masked thresholds for the sinusoidal test vibration)

The order of sessions in which the test frequencies were presented was randomised. Within each session, Part A was carried out at the start and was followed by Part B. Part B involved five intensities (i.e., 0 to $24 \mathrm{dBSL}$ in $6 \mathrm{~dB}$ steps) of the masker (i.e., $4-\mathrm{Hz}$ one-third octave bandwidth random vibration). The five different intensities of the masker were presented in a random order but with the restriction that the low-intensity maskers (i.e., 0 or $6 \mathrm{dBSL}$ ) were presented first and the high-intensity maskers (i.e., 12, 18, and $24 \mathrm{dBSL}$ ) were presented last. This procedure was adopted to minimise any cumulative effects of adaptation, such as might occur due to temporary threshold shifts (Gescheider and O'Malley, 1983).

A two-interval two-alternative forced-choice (2IFC) tracking method (Zwislocki et al., 1958) was employed for both Part A and Part B.

In Part A (i.e., unmasked thresholds of the sinusoidal test stimulus and thresholds for the random masker stimulus), subjects were presented with pairs of stimuli, each $2 \mathrm{~s}$ in duration, separated by a 1-s pause. An example sequence of stimuli in a trial is illustrated in Figure 2. The two observation periods were designated to the subjects by cue lights. The task of the subject was to judge whether the first or the second observation period contained a vibration stimulus. Subjects responded by saying "first" or "second".

In Part B (i.e., measurement of masked thresholds), a 2-s test stimulus was presented followed by a 1-s pause followed by a 7-s masker stimulus. The masker stimulus contained a set of two observation periods, each $2 \mathrm{~s}$ in duration, placed in the middle of the masker stimulus. An example sequence of stimuli in a trial is illustrated in Figure 2. Different cue 
lights were presented so as to distinguish the period when the test stimulus was initially presented from the observation periods; the light blinked during the period of a test stimulus, and the light was continuous during each of the two observation periods. The task of the subjects was to judge whether the first or the second observation period contained the test stimulus presented at the beginning of each trial. Subjects responded by saying "first" or "second".

The up-down transformed response (UDTR) procedure with a three-down one-up rule described by Wetherill and Levitt (1965) was employed to determine thresholds in both Part A and Part B. In both parts, the magnitude of the vibration stimulus was increased by $2.0 \mathrm{~dB}$ (a 25.8\% increment) after a negative (incorrect) response and decreased by $2.0 \mathrm{~dB}$ after three consecutive positive (correct) responses. This procedure provides thresholds corresponding to $79.4 \%$ correct responses. A trial was terminated after six reversals: a point where the stimulus level reversed direction (i.e., at either a peak or a trough). The threshold was calculated from the mean of the last two peaks and the last two troughs, omitting the first two reversals, as suggested by Levitt (1971).

During the experiment (both Part A and Part B), the subjects were seated comfortably with their backs supported by the backrest (see Figure 1). They were instructed to maintain their sitting postures with their hands on their laps, looking straight ahead. The experimenter adjusted the height of the footrest for individual subjects so as to ensure the upper surfaces of their upper legs were horizontal. 


\subsection{Analysis}

The amount of masking is expressed in decibels, taking the masked threshold with a masker presented at an intensity of $0 \mathrm{dBSL}$ with each subject as a reference:

$$
\text { Masked threshold }(\mathrm{dB})=20 \cdot \log _{10}\left(\frac{A_{\mathrm{NdB}}(f)}{A_{0 \mathrm{~dB}}(f)}\right)
$$

where, at frequency $f, A_{\mathrm{NdB}}(f)$ is the threshold (r.m.s. acceleration) with the masker at $N$ $\mathrm{dBSL}$, and $A_{0 \mathrm{~dB}}(f)$ is the threshold (r.m.s. acceleration) with the masker at $0 \mathrm{dBSL}$.

The magnitude of the sinusoidal test stimulus presented with the masker stimulus was calculated from the acceleration magnitude of the test vibration presented before the masker (see Part B in Figure 2).

Statistical analysis was performed using non-parametric tests, because it was assumed the measured thresholds would not be normally distributed: Friedman two-way analysis of variance for $k$-sample case and Wilcoxon matched-pairs signed ranks test for two-sample case. The criterion for statistical significance was set at $0.05(5 \%)$.

\section{RESULTS}

\subsection{Unmasked thresholds}

Individual and median thresholds for the perception of sinusoidal fore-and-aft vibration of the back at the four test frequencies $(4,8,16$, and $31.5 \mathrm{~Hz})$ are shown in Figure 3 . The lowest median acceleration thresholds (about $0.01 \mathrm{~ms}^{-2}$ r.m.s.) were obtained at 4 and $8 \mathrm{~Hz}$, with no significant differences between these frequencies (Wilcoxon, $p=0.26$ ). At frequencies from 8 to $31.5 \mathrm{~Hz}$, thresholds increased with each increase in frequency (Wilcoxon, $p<0.01$ ).

Median thresholds for the masker (one-third octave band centred on $4 \mathrm{~Hz}$ ) determined over the four exposures (one threshold obtained in each part) were about $0.01 \mathrm{~ms}^{-2}$ r.m.s. and similar to unmasked thresholds for $4-\mathrm{Hz}$ sinusoidal vibration (Figure 4). There were no 
significant differences in thresholds for the masker between the four exposures (Friedman, $p=0.25)$, indicating no obvious order effect.

FIG. 3 ABOUT HERE

FIG. 4 ABOUT HERE

\subsection{Masked thresholds}

Individual masking functions (threshold shifts caused by the 4-Hz random masker) for each frequency of sinusoidal vibration are shown in Figure 5 . The slopes obtained by linear regression for each test frequency show that the rate of increase in threshold with increasing level of the masker depended on the test frequency. With test frequencies of 4,8 , and $16 \mathrm{~Hz}$, each increase in the level of the masker increased thresholds, showing high correlations between the threshold shifts and the masker levels (Spearman, $p<0.01, r>0.65$ ). With the test frequency at $31.5 \mathrm{~Hz}$, the threshold shifts showed no correlation with the masker levels, indicating the $31.5-\mathrm{Hz}$ thresholds were little influenced by the $4-\mathrm{Hz}$ masker at any magnitude of vibration investigated (Spearman, $p=0.089, r=0.257$ ). The slopes of the linear regressions decreased with each increase in the test frequency (Figure 6; Wilcoxon, $p<0.015$ ).

FIG. 5 ABOUT HERE

FIG. 6 ABOUT HERE

\section{DISCUSSION}

The thresholds for fore-and-aft backrest vibration determined in the present study are similar in shape to thresholds determined with similar upright sitting postures by Basri and Griffin (2011) and Gallais et al. (2014), as overlaid in Figure 7. The greater median thresholds obtained by Basri and Griffin (2011) compared to the current results may be partly explained by the use of different psychophysical methods: absolute thresholds obtained with the 'forced-choice' procedure (employed in this study) tend to be lower than those obtained with 
the 'yes-no' procedure (employed by Basri and Griffin, 2011), consistent with the different criterion used when judging whether a stimulus is present in the two procedures, as demonstrated by Morioka and Griffin (2002). The median absolute thresholds have similar acceleration at 4 and $8 \mathrm{~Hz}$ and similar velocity at 8,16 , and $31.5 \mathrm{~Hz}$, consistent with the $W_{c}$ frequency weighting for fore-and-aft backrest vibration in the current British Standard (BS $6841,1987)$ and the current International Standard (ISO 2631-1, 1997). This suggests the $W_{c}$ weighting is appropriate for evaluating vibration with respect to vibration perception as well as vibration discomfort.

The masking provided by the random vibration centred on $4 \mathrm{~Hz}$ depended on the frequency of the sinusoidal test stimulus: the threshold shift reduced as the difference in frequency between the test stimulus and the masker stimulus increased. As summarised in Figure 8, the slopes of the masking functions decreased by about $0.3 \mathrm{~dB} / \mathrm{dB}$ with each doubling of the test frequency.

\section{FIG. 8 ABOUT HERE}

A slope of $1.0 \mathrm{~dB} / \mathrm{dB}$ in a masking function indicates that a vibration stimulus will not be detected if the background vibration has the same (or greater) intensity. The sinusoidal $4-\mathrm{Hz}$ test stimuli were almost completely masked by the random masker centred on $4 \mathrm{~Hz}$ (slope of 0.9). It is reasonable to assume that masking occurred because the $4-\mathrm{Hz}$ sinusoidal test motion and the $4-\mathrm{Hz}$ random masker were detected by the same sensory system, or at the same body location. The $8-\mathrm{Hz}$ and the $16-\mathrm{Hz}$ test stimuli were partly masked by the $4-\mathrm{Hz}$ masker (slopes of 0.6 and 0.3 , respectively). This partial masking may partly be due to some overlap of body locations involved in the perception of the test stimuli and the masker stimulus. With the $31.5-\mathrm{Hz}$ vibration, there was little masking with any intensity of the $4 \mathrm{~Hz}$ masker (slope of 0.1).

Most subjects reported they detected the $4-\mathrm{Hz}$ vibration at the back in the skin near the vibrating backrest, whereas they tended to perceive the 8 - and $16-\mathrm{Hz}$ vibrations at other 
body locations in addition to the back (i.e., at the back, neck, shoulder, and chest). Subjects said the $31.5-\mathrm{Hz}$ vibration was mostly perceived within the body between the back and the chest, unlike the sensations produced by the $4 \mathrm{~Hz}$ vibration. With a wider range of vibratory stimuli, vibration might be felt at almost any location in the body or detected by sight or hearing.

It seems reasonable to suppose that similar masking to that found here will occur in the converse situation with the test and the masker exchanged: so the detection of a $4-\mathrm{Hz}$ 'event' in a vehicle (e.g., a transient fore-and-aft motion when a car changes gear) will depend on the characteristics of the background vibration. In the extreme, the perception of such a transient will be prevented by the presence of a $4-\mathrm{Hz}$ background vibration with a similar magnitude, but perception will be unimpeded by a $31.5-\mathrm{Hz}$ background vibration of any magnitude.

It is likely that masking similar to that observed in this study with fore-and-aft backrest vibration will also occur with other directions and other locations of contact with the vibration of a seat. However, masking functions will differ from those found here because the frequency-dependence of the location of perceiving vibration within the body varies with the direction and the location of excitation (Whitham and Griffin, 1978). With suitable additional measurements of masked thresholds it should be possible to develop a family of models of masking using masking functions similar to those determined in the present study.

The overall severity of a vibration (i.e., whether it can be perceived or how uncomfortable it feels) is currently predicted using frequency weightings to 'weight' the vibration acceleration according to human sensitivity. Even if this predicts the overall severity of the vibration, the existence of masking means that the perception of a specific vibration component may not be well predicted from its mathematical contribution to the overall r.m.s. magnitude of the frequency-weighted vibration. 
The findings of this study seem consistent with masking primarily arising when vibration is felt in the same location of the body. It seems possible that two different mechanisms may be involved, with masking being dependent on whether the sensations are within the same sensory system and at the same location. However, depending on the definition of a 'channel', a separation in location may result in perception occurring via separate sensory systems (e.g. one vibration stimulus perceived by a somatosensory system and another vibration stimulus perceived by the vestibular or visual system). Further research on the means of perceiving sensations arising from whole-body vibration is required to identify and model the mechanisms involved in producing overall sensations and the masking of vibratory stimuli. Understanding masking associated with whole-body vibration should help to improve vehicle ride quality by making it possible to predict whether a particular vibration will be detected by drivers or passengers when there are other vibrations present.

\section{CONCLUSIONS}

Absolute thresholds for the perception of fore-and-aft vibration of a backrest depend on the frequency of vibration, with similar acceleration thresholds from 4 to $8 \mathrm{~Hz}$ and similar velocity thresholds from 8 to $31.5 \mathrm{~Hz}$. Absolute thresholds for perceiving fore-and-aft backrest vibration have a similar frequency-dependence to equivalent comfort contours for backrest vibration, so the same frequency weighting can be used to predict the discomfort and the perceptibility of fore-and-aft vibration of backrests.

The threshold for perceiving backrest vibration at one frequency can be 'masked' by another vibration with either the same frequency or a different frequency, with the 'threshold shift' reducing with increasing difference between the frequencies of the two vibrations. A narrow band of random vibration centred on $4-\mathrm{Hz}$ will almost completely mask the perception of 4$\mathrm{Hz}$ sinusoidal backrest vibration, partially mask 8- and $16-\mathrm{Hz}$ backrest vibration, but provide little masking of $31.5-\mathrm{Hz}$ backrest vibration. The masking might be explained by the 
involvement of different sensory systems (e.g. channels of information within the somatosensory, vestibular, visual systems) and different body locations in the perception of vibratory stimuli at different frequencies.

\section{ACKNOWLEDGEMENT}

This research was funded by Jaguar Land Rover Ltd. The authors would like to acknowledge the contributions of Mr. Neil Dixon for his advice and kind support throughout the work. 


\section{REFERENCES}

Basri B, and Griffin MJ (2011) The vibration of inclined backrests: perception and discomfort of vibration applied normal to the back in the x-axis of the body. Journal of Sound and Vibration 330, 4646-4659.

Basri B, and Griffin MJ (2014) The application of SEAT values for predicting how compliant seats with backrests influence vibration discomfort. Applied Ergonomics, 45, 1461-1474.

British Standards Institution (1987) Measurement and evaluation of human exposure to whole-body mechanical vibration and repeated shock. BS 6841

Gallais CHR, Morioka M, and Griffin MJ, Thresholds for the perception of fore-and-aft wholebody-vibration. 2015 (awaiting publication).

Gescheider GA, Verrillo RT, and van Doren CL (1982) Prediction of vibrotactile masking functions. The Journal of the Acoustical Society of America 72: 1421-1426.

Gescheider GA, O'Malley MJ, and Verrillo RT (1983) Vibrotactile forward masking: evidence for channel independence. The Journal of the Acoustical Society of America 74(2):474-485.

International Organization for Standardization (1997) Mechanical vibration and shock Evaluation of human exposure to whole-body vibration - Part 1: General requirements. ISO 2631-1, Geneva.

Jalil NAA, and MJ Griffin (2007) Fore-and-aft transmissibility of backrests: variation in transmissibility with the height above the seat surface and non-linearity. Journal of Sound and Vibration 299, 109-122

Jalil NAA, and MJ Griffin (2008) Fore-and-aft apparent mass of the back: Nonlinearity and variation with vertical location. Journal of Sound and Vibration 318, 1348-1363.

Kato K, and Hanai T (1998) The effect of backrest angles on discomfort caused by fore-andaft back vibration. Industrial Health 36, 107-111.

Levitt H (1971) Transformed Up-Down Methods in Psychoacoustics. The Journal of the Acoustical Society of America 49, 467-477.

Morioka M, and Griffin MJ (2002) Dependence of vibrotactile thresholds on the psychophysical measurement method. Int Arch Occup Environ Health 75, 78-84.

Morioka M, and Griffin MJ (2005) Independent responses of Pacinian and Non-Pacinian systems with hand-transmitted vibration detected from masked thresholds. Somatosensory and Motor Research 22 (1/2), 69-84.

Morioka M, and Griffin MJ (2010) Frequency weightings for fore-and-aft vibration at the back: effect of contact location, contact area, and body posture. Industrial Health 48, 538-549.

Durlach NI, Mason CR, Kidd G, Arbogast TL, Colburn HS, and Shinn-Cunningham BG (2003) Journal of Acoustical Society of America 113(6), 2984-2987.

Parsons KC, and Griffin MJ and Whitham EM (1982) Vibration and comfort III. Translational vibration of the feet and back. Ergonomics 25, 705-719.

Wetherill GB, and Levitt $H$ (1965) Sequential estimation of points on a psychometric function. The British Journal of Mathematical and Statistical Psychology 18: 1-10.

Whitham EM, and Griffin MJ (1978) The effects of vibration frequency and direction on the location of areas of discomfort caused by whole-body vibration. Applied Ergonomics, 9,(4), 231-239.

Zwislocki J, Meire F, Feldman AS, and Rubin H (1958) On the effect of practice and motivation on the threshold of audibility. The Journal of the Acoustical Society of America 30: 254-262. 


\section{FIGURE LEGENDS}

FIG. 1 Posture adopted by the subjects sitting on a stationary rigid seat with a stationary footrest and exposed to fore-and-aft vibration at the back. The subjects maintained their sitting postures with their hands on their laps, looking straight ahead.

FIG. 2 Stimulus timing of a trial for Part A (unmasked threshold) and Part B (masked threshold). Part B example illustrates a $1 / 3$-octave bandwidth masker centred on $4 \mathrm{~Hz}$ with a test stimulus of $8 \mathrm{~Hz}$ occurring during the second observation period.

FIG. 3. Unmasked thresholds from nine subjects overlaid with median data.

FIG. 4. Thresholds of the masker (4-Hz 1/3-octave bandwidth random vibration) for nine subjects and the median thresholds over four exposure periods (thresholds obtained in each of the four sessions).

FIG. 5. Individual masking functions for each test frequency and slopes of linear regressions of the thresholds from nine subjects.

FIG. 6. Slopes of masking functions for the nine subjects overlaid with median slopes for each of the four frequencies. Dotted line presents linear regressions of the median slopes with the equation.

FIG. 7. Median unmasked thresholds overlaid with median thresholds from Basri and Griffin (2011) and Gallais et al. (2015) and reciprocal of $W_{c}$ weighting (normalised to $0.01 \mathrm{~ms}^{-2}$ r.m.s. at $1 \mathrm{~Hz})$.

FIG. 8 Masking functions for sinusoidal test frequencies of $4,8,16$, and $31.5 \mathrm{~Hz}$ with $4-\mathrm{Hz}$ masker vibration. 


\section{TABLE LEGENDS}

Table 1. Stimulus characteristics of the test stimulus and the masker. 
FIG. 1 Posture adopted by the subjects sitting on a stationary rigid seat with a stationary footrest and exposed to fore-and-aft vibration at the back. The subjects maintained their sitting postures with their hands on their laps, looking straight ahead.

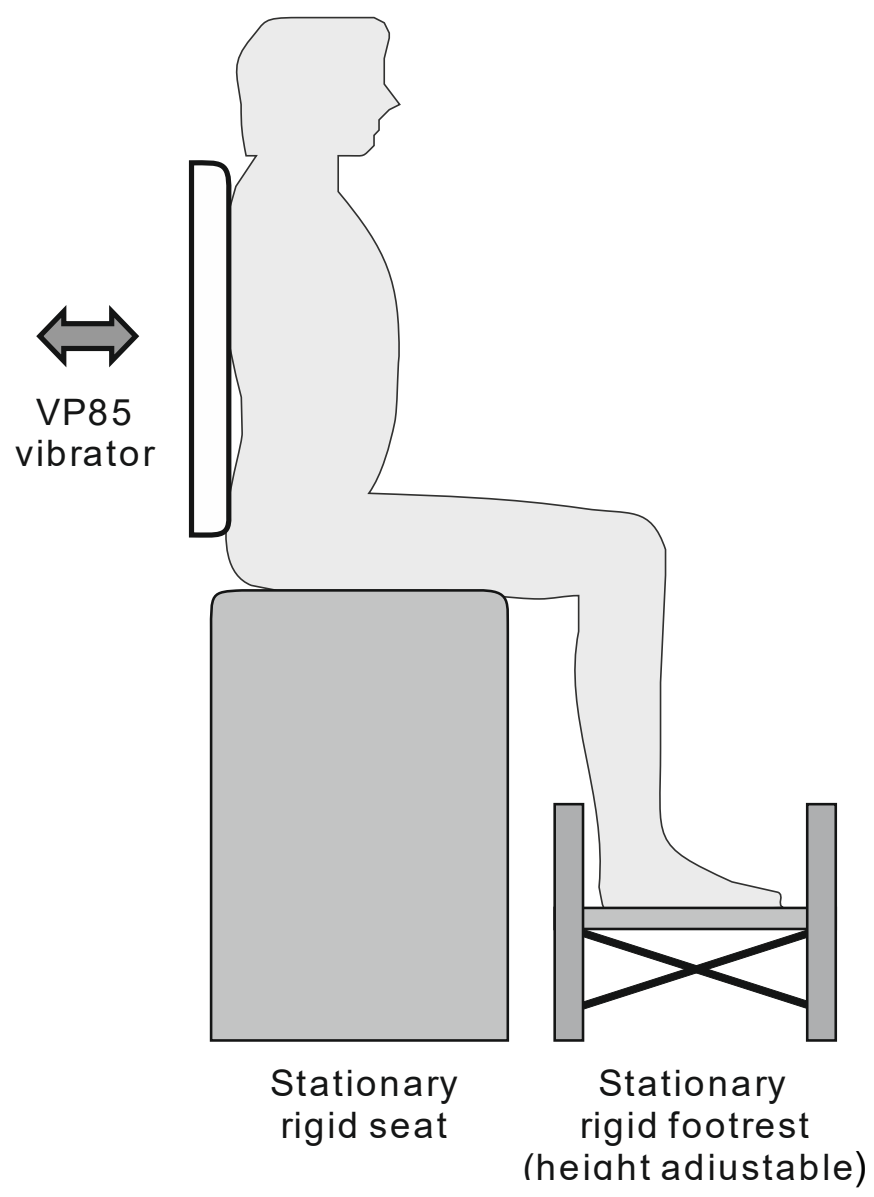


FIG. 2 Stimulus timing of a trial for Part A (unmasked threshold) and Part B (masked threshold). Part B example illustrates a $1 / 3$-octave bandwidth masker centred on $4 \mathrm{~Hz}$ with a test stimulus of $8 \mathrm{~Hz}$ occurring during the second observation period.

Part A: Unmasked thresholds

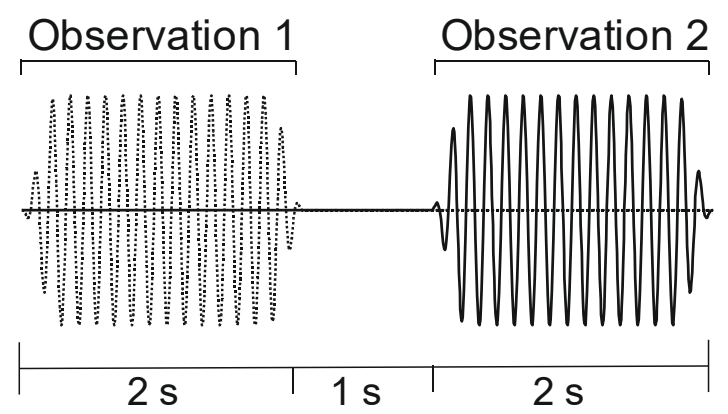

Part B: Masked thresholds

Masker

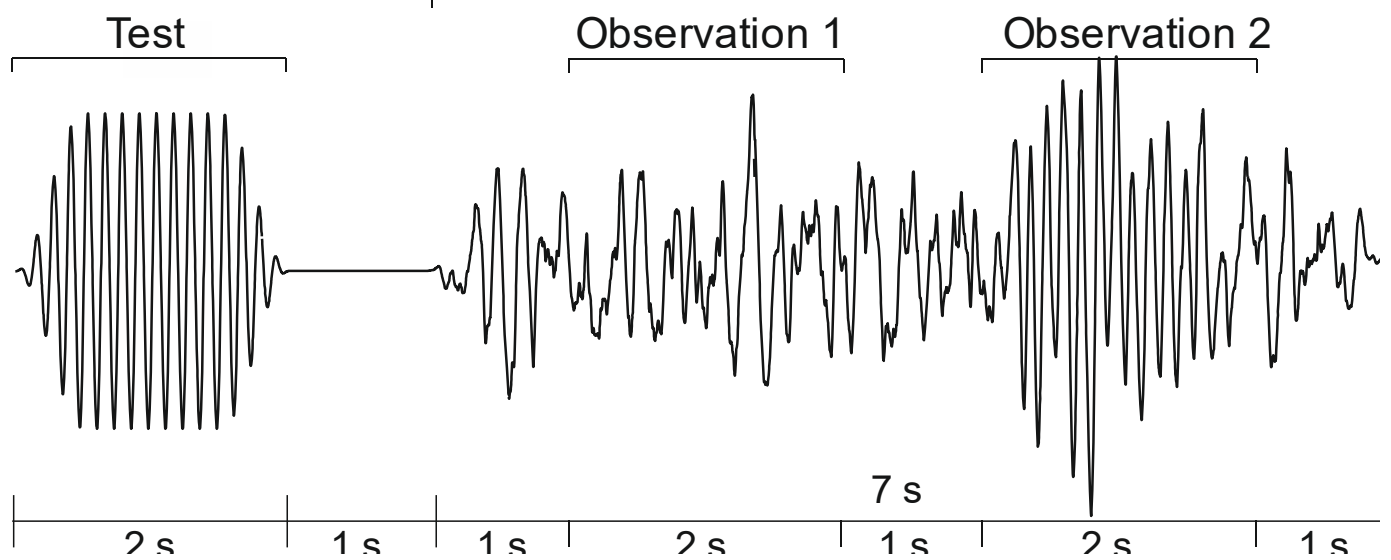


Published as: Morioka, M. and Griffin, M.J (2015).

Masking of thresholds for the perception of fore-and-aft vibration of seat backrests Applied Ergonomics. 50, p. 200-206.

FIG. 3. Unmasked thresholds from nine subjects overlaid with median data.

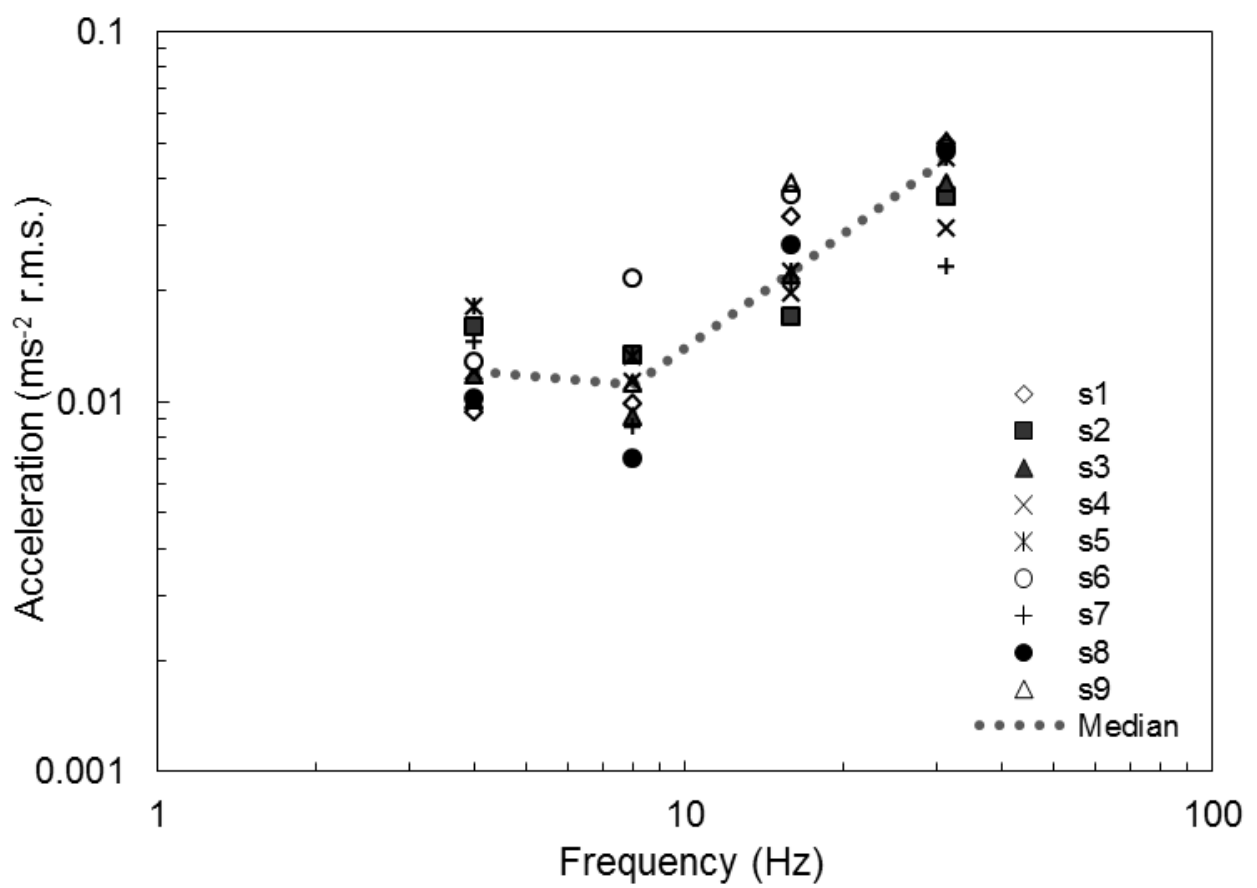


FIG. 4. Thresholds of the masker (4-Hz 1/3-octave bandwidth random vibration) for nine subjects and the median thresholds over four exposure periods (thresholds obtained in each of the four sessions).

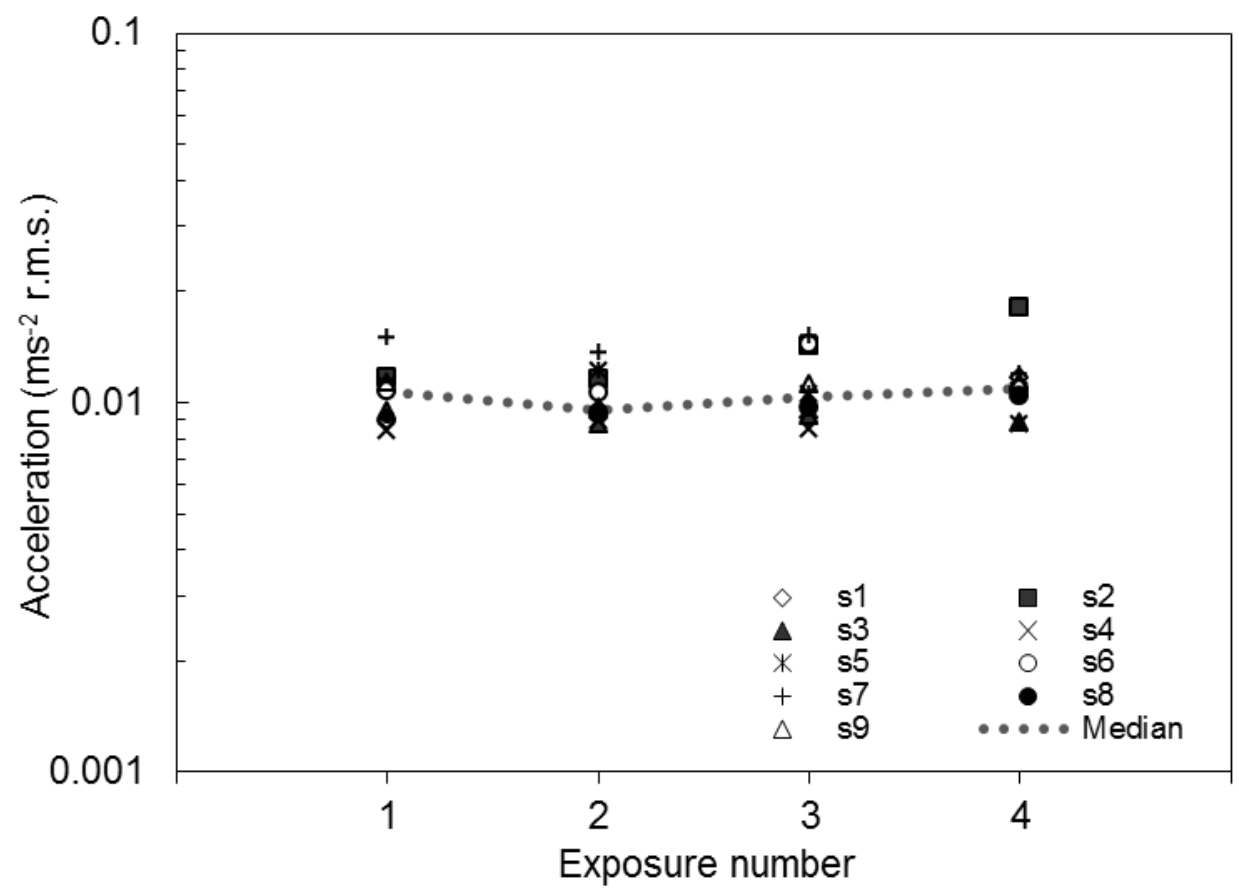


FIG. 5. Individual masking functions for each test frequency and slopes of linear regression of the individual thresholds from nine subjects.
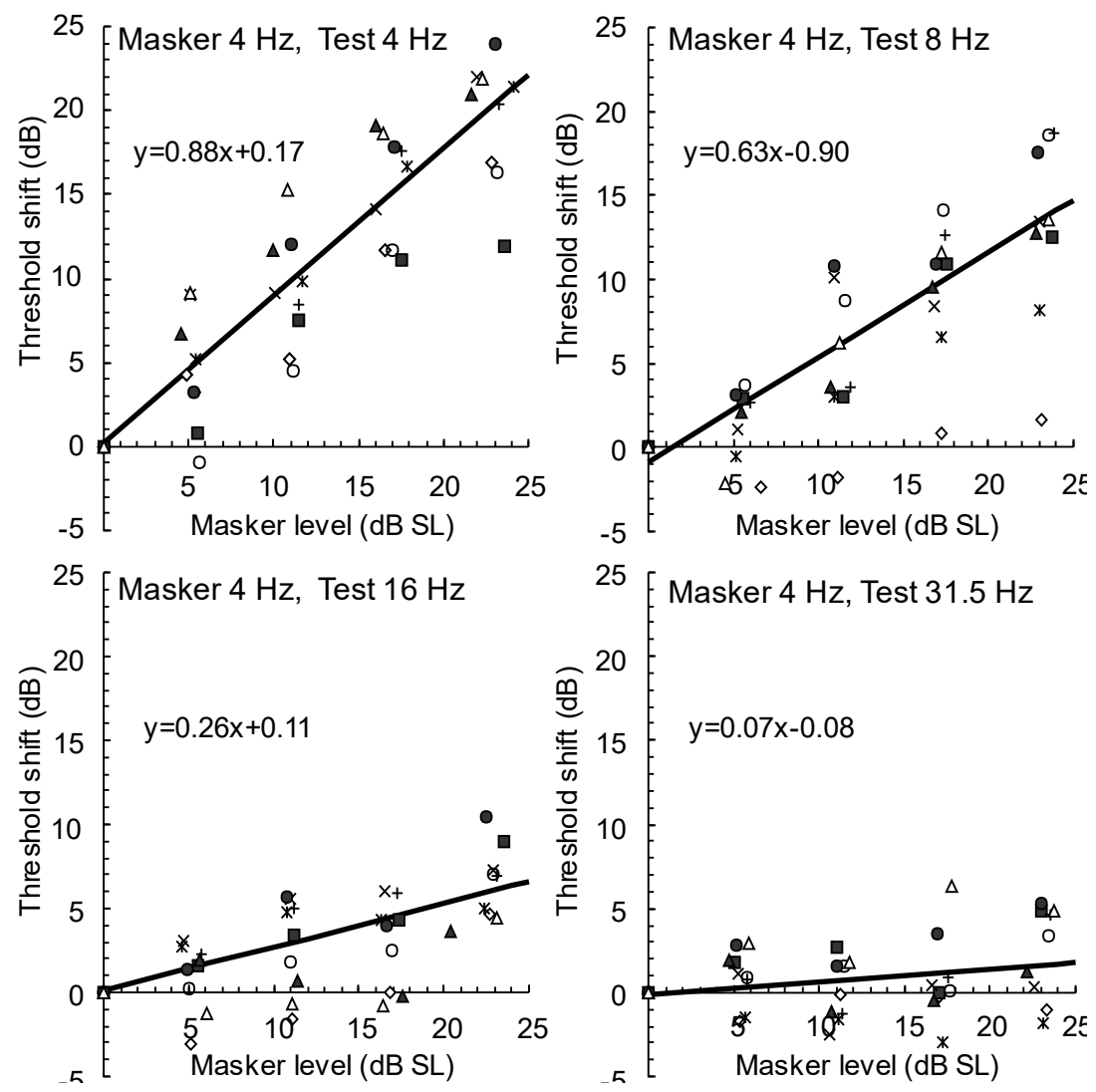
FIG. 6. Slopes of masking functions for the nine subjects overlaid with median slopes for each of the four frequencies. Dotted line presents linear regressions of the median slopes with the equation.

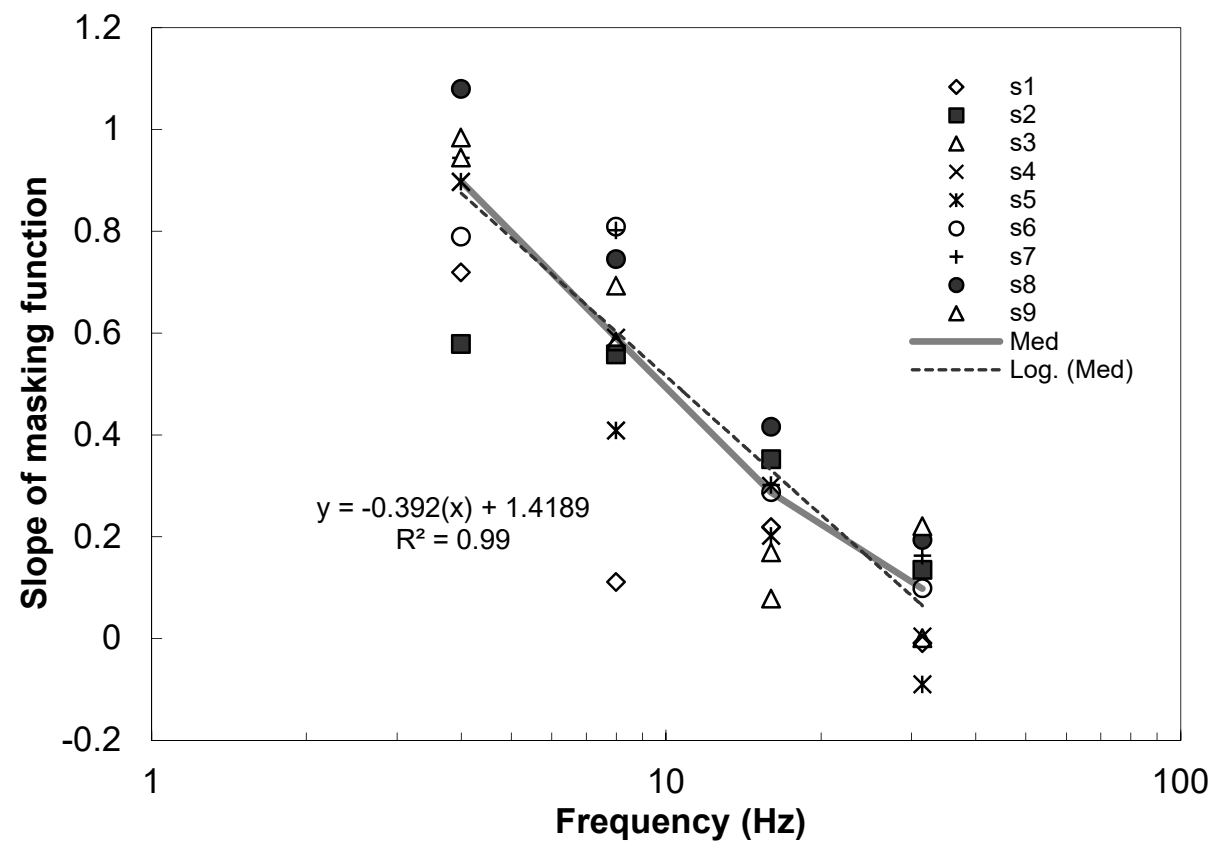


FIG. 7. Median unmasked thresholds overlaid with median thresholds from Basri and Griffin (2011) and Gallais et al. (2015) and reciprocal of $W_{c}$ weighting (normalised to $0.01 \mathrm{~ms}^{-2}$ r.m.s. at $1 \mathrm{~Hz})$

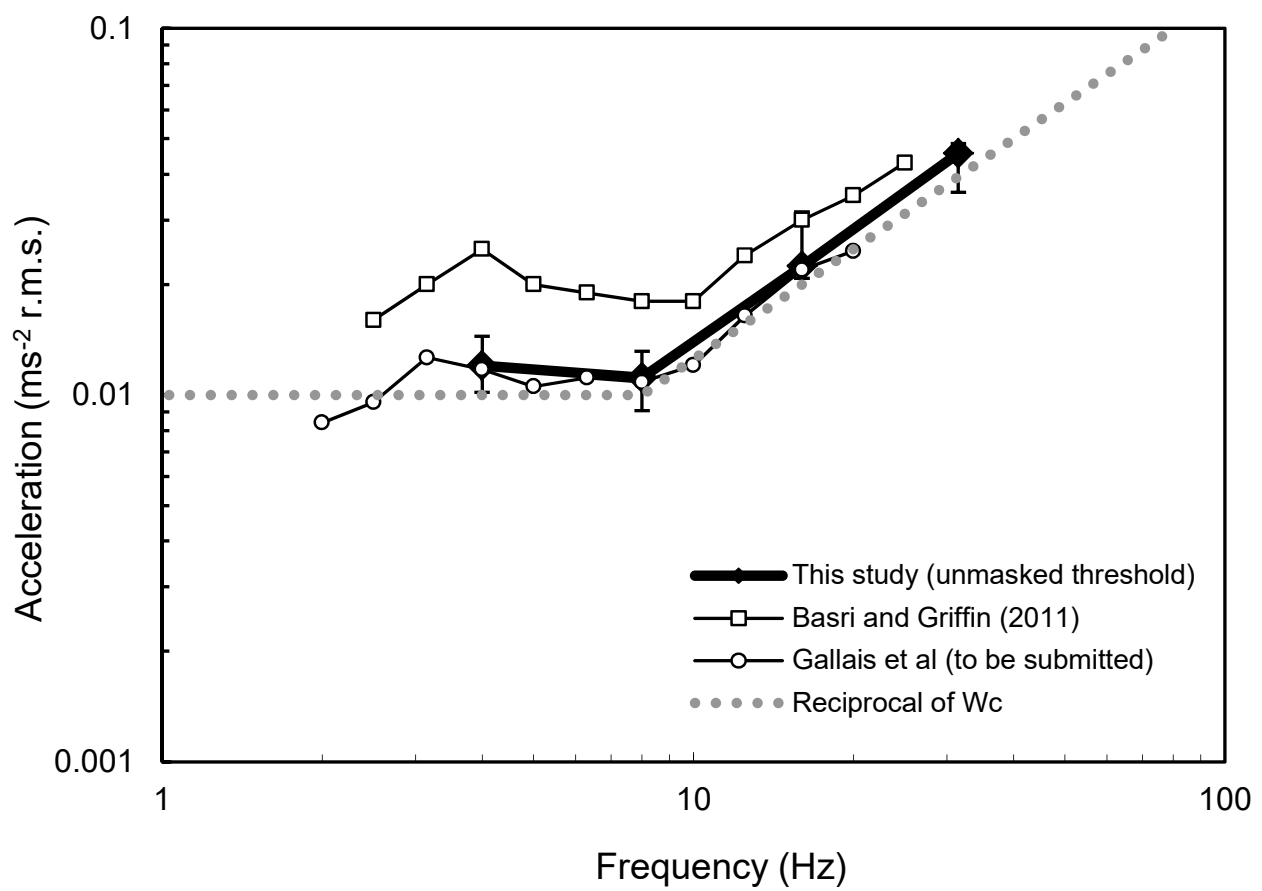


FIG. 8. Masking functions for sinusoidal test frequencies of $4,8,16$, and $31.5 \mathrm{~Hz}$ with $4-\mathrm{Hz}$ masker vibration.

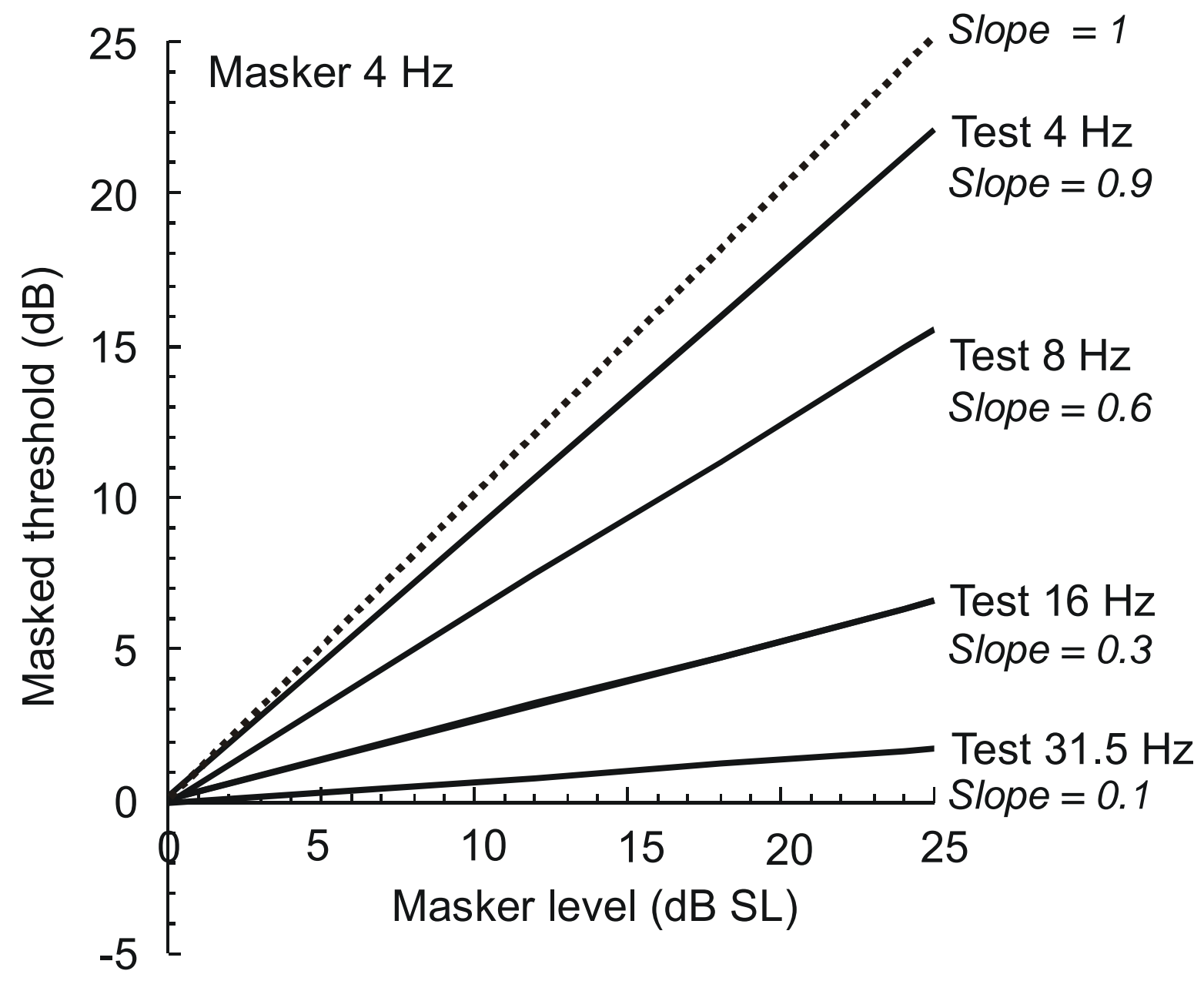


Table 1 Stimulus characteristics of the test stimulus and the masker presented over four sessions in Part B.

\begin{tabular}{|c|c|c|}
\hline Session & Test stimulus & Masker stimulus \\
\hline a & $4 \mathrm{~Hz}$ & \multirow{2}{*}{$\begin{array}{r}\text { 4 Hz centred } 1 / 3 \text { octave bandwidth vibration } \\
\text { at } 0,6,12,18 \text { and } 24 \mathrm{dBSL}^{*}\end{array}$} \\
\cline { 1 - 2 } $\mathrm{b}$ & $8 \mathrm{~Hz}$ & \\
\hline $\mathrm{c}$ & $16 \mathrm{~Hz}$ & \\
\hline $\mathrm{d}$ & $31.5 \mathrm{~Hz}$ & \\
\hline
\end{tabular}

${ }^{*} \mathrm{dBSL}=$ decibels in intensity of vibration relative to the absolute threshold of the masker determined in Part A. 